\title{
Sentiments perçus quant au choix de la discipline du football
}

\section{Résumé}

L'étude porte sur une population de 190 footballeurs Algériens, tous signataires dans des clubs sportifs évoluant en ligue1. Le but est de déterminer les sentiments perçus quant au choix de la discipline du football. Pour ce faire, nous avons choisi comme protocole expérimental, le questionnaire de type Likert en utilisant des items catégoriels bipolaires à deux dimensions, motivation personnelle et milieu sportif. Les résultats auxquels nous sommes arrivés soulignent qu'il est impossible aujourd'hui de choisir la discipline du football sans un environnement social favorable et un milieu sportif adéquat.

Mots clefs : football, choix, sentiment perçu, environnement social, milieu sportif

\section{Farida MOKRAN \\ Faïza GOUMIRI \\ Ahcène KASMI}

Institut d'Education Physique et Sportive Université d'Alger 3

Laboratoire des Sciences du Sport et de l'Entrainement de Haut Niveau (Algérie)

\section{Introduction}

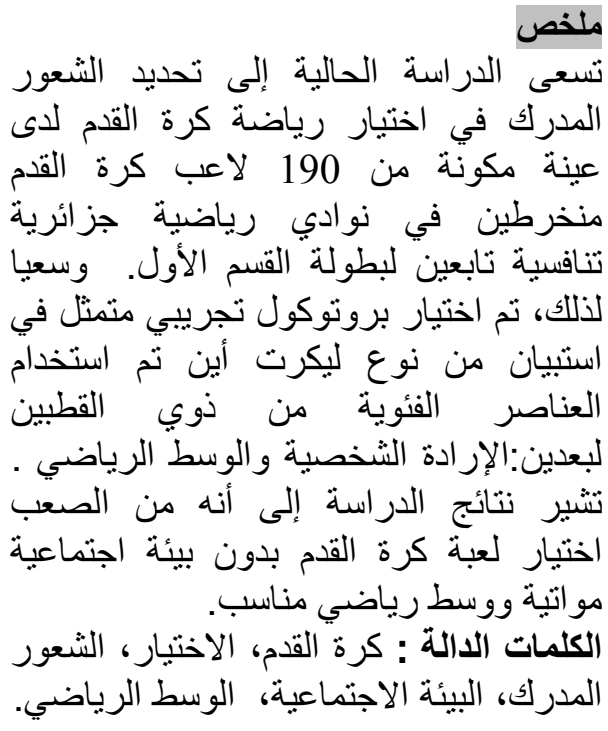

Le sujet que l'on se propose de traiter consiste en la détermination de sentiments perçus quant au choix de la discipline du football, sachant que la pratique de cette discipline ne peut être un fait du hasard. Cette difficulté s'explique par le fait que, depuis la naissance de cette discipline en Chine, ce sport ne cesse d'évoluer. Il est passé d'une structure classique, qui est à la portée de tous, à une structure moderne avec ses différentes formes d'exigences. La littérature spécialisée précise qu'il est difficile aujourd'hui de se faire une place dans cette discipline (1). N'avons- nous pas déjà entendu de la part des parents, ou même des techniciens du sport à propos du devenir d'un enfant que ce dernier ferait entendre parler de lui. Cependant, il s'avère que 
quelques années après, certains de ces enfants sont retenus, alors que d'autres sont écartés ou bien abandonnent.

C'est ainsi que la question du choix de la discipline du choix du football se pose. Même si les raisons sont diverses, elles convergent vers des travaux (2) qui mettent en évidence l'environnement social et le milieu sportif qui auront obligatoirement une incidence sur son comportement décisionnel quelque soit le niveau de pratique et la discipline sportive.

\section{Méthodologie}

\section{- But de l'étude}

Le but de cette étude est déterminé dans les détails les plus précis à propos des sentiments perçus quant au choix de la discipline du football.

\section{- Population d'étude}

Nous avons interrogé 190 footballeurs de la catégorie des U20 $(\mathrm{SD}=1.02)$ évoluant en ligue1. Ces joueurs avaient une expérience de sept années de pratique en moyenne $(\mathrm{SD}=0.94)$ dans le domaine de la compétition.

\section{- Procédure}

Un questionnaire utilisant une échelle de Likert à items catégoriels bipolaires a été choisi comme outil d'investigation. Ce questionnaire est inspiré du modèle établit par Vallerand et coll. (3) dans le cadre de leurs travaux sur les différentes formes de motivation développées par les participants vis-à-vis de l'activité sportive pratiquée. Cette technique du questionnaire a été reprise sous une forme abrégée dans le cadre d'une étude sur l'engagement et le désengagement de la pratique sportive, en utilisant des items catégoriels bipolaires qualifié de question à choix multiple(4).

Le questionnaire est réparti en deux dimensions: l'une ayant trait à l'environnement social du footballeur; l'autre au milieu sportif. La passation du questionnaire s'est faite durant la phase aller de la période compétitive, juste avant le début de la séance d'entrainement afin d'éviter toute perturbation du programme de l'entraineur.

L'environnement social est traité en trois items dont les questions tournent autour $\mathrm{du}$ « statut social du footballeur, l'argent et la célébrité ». Le milieu sportif est traité en 6 items dont les questions se limitent autour des éléments tels que « le sentiment d'indépendance, la sensation de plaisir, la coopération, la simplicité de jouer au football, le rôle de l'enseignant de l'EPS, du dirigeant de club, de l'entraineur ainsi que les infrastructures sportives ».

\section{- Analyse Statistique}

Les résultats obtenus ont été intégrés dans une base de données et traités au moyen du logiciel statistique des sciences sociales (SPSS). Le test du Khi-deux a permis de dresser des tables de fréquences et des tableaux de tris croisés en vue de comparer les résultats obtenus. 


\section{Résultats et discussion}

\section{- Le statut de footballeur de haut niveau}

Les résultats statistiques dénotent une différence significative en faveur des réponses $(70 \%)$ «Plutôt d'accord» sur la recherche du statut du footballeur "J'ai vraiment envie de devenir un footballeur professionnel, je veux évoluer au haut niveau ».

A priori, la réponse la plus proche à ce questionnement est sans doute liée à la professionnalisation et à la commercialisation de cette discipline. Ces changements ne peuvent laisser indifférents les principaux acteurs (footballeurs) et leurs dirigeants. Il semble être légitime pour eux de faire valoir leurs droits. Ainsi, le statut de footballeur professionnel est d'avoir un contrat de travail avec indemnités comprises. Ce statut est différent de celui du fonctionnaire (5). Il est clairement plus avantageux et varie selon le niveau de compétition auquel il appartient, et dans lequel il évolue (6).

Dans tous les cas de figure, le joueur sera lié à un club employeur. Il doit consacrer une plus grande partie de son activité et de son temps à la pratique du football. Une présence journalière voire même, plusieurs heures sont nécessaires pour l'entrainement et les compétitions. La distinction de ce footballeur est représentée par son engagement pour un certain nombre d'années de pratique du football de haut niveau sans aucune trêve.

Durant sa carrière, il doit respecter un mode de vie spécifique à savoir respecter une alimentation saine, des temps de sommeil suffisants afin d'assurer une meilleure récupération. Il doit également participer aux entrainements physiques, technicotactiques, suivre une préparation psychologique régulière ainsi que des contrôles médico-sportifs. Autrement dit, ces sacrifices sont à consentir pour pouvoir accéder au statut de footballeur professionnel.

\section{- L'argent}

Les données statistiques soulignent une différence significative en faveur des réponses (76\%) «Assez d'accord» concernant la recherche de l'argent. La question que l'on peut se poser est de voir si l'on peut trouver de l'argent en football.

Plusieurs éléments de réponses peuvent être accordés à ce type d'interrogation telle que l'introduction de l'argent dans le domaine du football, malgré la résistance de $\mathrm{P}$. Coubertin contre le professionnalisme du sport; sachant que les premières transactions ont commencés dès 1884, lorsqu'un roi du coton de Preston (Angleterre) avait fait scandale en engageant contre de l'argent les meilleurs footballeurs du pays (7).

L'arrivée massive de l'argent s'explique par la médiatisation de cette discipline. Les sportifs sont considérés comme « marchandise » qui permet de produire de l'argent, de construire des images et de les vendre. Certains sportifs gagnent en trois mois ce qu'un salarié moyen ne touchera pas durant toute sa vie (8). Le football est à l'heure actuelle une institution bureaucratique, dotée d'une structure totalement déterminée par le mode de production capitaliste (9).

Cette ouverture au marché économique s'est traduite par la multiplication des clubs professionnels qui connaissent actuellement la logique de l'offre et de la demande. 
Les grands clubs achètent systématiquement les meilleurs joueurs. Leur engagement et leur transfert entre les clubs sont définis par des transactions financières importantes et légales. Ces joueurs bénéficient des sommes hors normes comme l'indique la grille de rémunération des joueurs de football.

\section{- La célébrité}

Les calculs statistiques évoquent une différence significative en faveur des réponses (89\%) «Tout à fait d'accord 》 sur la recherche de la célébrité. En d'autres termes, devenir célèbre est le rêve de tous les footballeurs. Cette discipline réunit les critères comme ceux du cinéma, qui en avaient fait un phénomène au siècle dernier. Le football est devenu donc, le moyen le plus simple pour atteindre la célébrité, il faut juste savoir bien jouer (tirer, dribbler, passer, marquer des buts...). Les audiences record lui donnent de l'importance et rendent son spectacle plus attrayant. C'est grâce à son talent sportif, que l'on peut devenir célèbre. Même dans les pays déjà fortement foot balisés, beaucoup de jeunes souhaitent devenir footballeurs. Cette discipline a su se faire une révolution en devenant le sport le mieux pratiqué au monde (10).

\section{- La place des études}

Les résultats statistiques dénotent une différence significative en faveur des réponses (74\%) "Plutôt d'accord » que l'on peut quitter les bancs de l'école pour rejoindre les terrains de football, ne semble pas être une position raisonnable, sachant que les études sont un investissement certain pour l'avenir.

Opter uniquement pour la pratique du football de haut niveau est un choix que la majorité des sportifs font, surtout lorsqu'il s'agit d'athlètes qui possèdent d'importantes prédispositions sportives.

Dans une interview adressée à une athlète de l'équipe nationale algérienne, «Mes études se sont très bien passées, sauf en terminal où j'ai eu des problèmes : c'était l'année des jeux olympiques de Barcelone (11). Il y avait une préparation difficile, donc, je n'arriverais pas à suivre les deux camps. Je n'ai pas passé mon baccalauréat cette année là, j'ai préféré de loin les J.O, l'examen du baccalauréat n'a pas été une de mes priorités, d'autant plus, que les programmes d'entrainement et de compétition ne m'ont pas laissé assez de temps pour m'occuper de mes études ». Sachant que la haute performance sportive exige à l'athlète des séances d'entrainements de plusieurs heures, voire 4 à 5 heures, par jour (12). La même athlète poursuit « Je n'ai jamais rencontré d'athlète qui réussit, aux études et au sport de haut niveau en même temps. On nous demande toujours de choisir ».

Une autre position, toute aussi importante concernant le délaissement des études au détriment de la pratique du football de haut niveau est intimement liée à l'incitation financière. Il est possible de gagner rapidement beaucoup d'argent, ce qui annonce une meilleure vie économique, surtout si ces sportifs proviennent de familles à faible revenu (13). Pourquoi perdre son temps et son énergie à étudier, puisque il est possible de gagner assez d'argent au football! 


\section{- Le sentiment d'indépendance}

Les données statistiques soulignent une différence significative en faveur des réponses (73\%) "Plutôt d'accord" sur le sentiment d'indépendance au cours de la pratique du football. On peut qualifier cette indépendance d'intense émotion de liberté.

Se sentir indépendant, est à notre avis, avoir la sensation de se défaire d'une domination, d'un contrôle exercé par un ou des tiers, ou par un système. Ce sentiment ne se ressent pas sans peine, puisqu'il s'agit de se libérer de pressions frustrantes qui sont à l'origine d'un manque de liberté. D'ou « le désir d'être soi, de se surpasser ».

L'indépendance du footballeur se manifeste à travers la maîtrise parfaite de son propre corps, du ballon et de l'adversaire ainsi qu'une capacité à agir positivement dans les différentes situations de jeu en fonction de l'adversaire.

Cette qualité s'acquiert par le développement de sa motricité et de sa perception, de même que par sa capacité à maintenir et à renouveler ses énergies afin d'assurer le maintien du haut niveau de sa capacité physique et psychologique.

Ce facteur d'indépendance entraine une importante disponibilité du joueur, devenant par la suite source de liberté d'action, garantissant l'ouverture à son entourage, en exerçant des choix créatifs et responsables. Grehaigne, y accordent à ce facteur une place considérable (14).

L'exemple le plus frappant de type de situation revient à Mercier, qui estime que le football de haut niveau a beaucoup évolué(15). Les permutations des joueurs, les montées répétées des arrières, les incursions des demis jusqu'à la position de tir, le rôle déterminant des attaquants dans la zone d'attaque et le soutien perpétuel du milieu et des défenseurs se limitent particulièrement aux simples initiatives du joueur. On doit alors croire à une grande marge d'indépendance du joueur.

\section{- La sensation de plaisir}

Les résultats statistiques évoquent une différence significative en faveur des réponses (78\%) "Assez d'accord " sur la sensation du plaisir dans la discipline du football. Quelle explication allons-nous donner à cette interrogation?

En effet, prendre du plaisir « est un état émotionnel agréable, une réponse affective positive vis-à-vis de la pratique sportive $»(2)$. Cet état est la résultante de multiples affects déterminés par divers éléments de la situation vécue par le sujet. La maîtrise de la discipline du football apporte à notre avis la contribution la plus significative de la sensation du plaisir. Ceci signifie que le coach doit faire en sorte que les séances d'entrainement qui sont souvent présentées comme difficiles, dures, stressantes, doivent plutôt prétendre à une forme de plaisir et non une contrainte (16).

Il doit constamment rappeler à ses joueurs les raisons de leurs investissements sportifs comme: le bonheur de progresser, l'acquisition des compétences, l'amélioration, la remise en question perpétuelle, le fait de se lancer des défis ainsi que la préparation des victoires futures. Ce n'est que dans ces conditions que l'entraineur peut transformer les difficultés de cette activité en plaisir de participer (entraînement et compétition). 


\section{- La coopération}

Les données statistiques dénotent une différence significative en faveur des réponses (74\%) "Plutôt d'accord" sur le phénomène de coopération que l'on trouve dans la discipline du football. Ce qui laisse entendre que ce type de sensation n'est pas un fait du hasard. Cette notion est abordée bien avant, dans les écrits de Taylor (17). Elle est à la base de toute organisation, dans la mesure où il est impossible pour une personne de réaliser un projet collectif sans qu'elle ne soit assistée. Ce qui signifie que la coopération, est à notre sens une attitude consciente et délibérée dans la pratique football.

Le football étant un sport collectif, au sein duquel chacun des joueurs est responsable de son compartiment ou encore de son poste jeu et ainsi accorde une place particulière au sentiment de coopération.

En bref, la coopération est dite forte, lorsque tous les membres de l'équipe coopèrent pour la progression du ballon de son propre camp à celui de l'équipe adverse sans commettre de fautes ou encore lorsque chacun se montre performant avec ses coéquipiers en se joignant à eux pour la récupération de la balle pour les fautes commises au cours du jeu. Il s'agit de travailler ensemble, d'agir conjointement pour aboutir à un objectif, où les tâches et les buts sont communs(18). Vermeulen (19) auteur de plusieurs publications dans le domaine du football, souligne: «qu'une équipe est idéale, c'est là où règne une parfaite coopération du collectif avec un total engagement individuel vers une vision commune ». Chaque joueur de l'équipe deviendra le coach de l'autre et aidera ses coéquipiers à remplir convenablement leur mission du collectif.

Chacun doit chercher l'excellence dans ses actions et être au service de l'équipe. La réussite d'une équipe dépend du bon équilibre entre l'individuel et le groupe.

\section{- La simplicité de jouer au football}

Les résultats statistiques évoquent une différence significative en faveur des réponses $(91 \%)$ "Tout à fait d'accord » quant à la simplicité de jouer au football. Les réponses à ces interrogations trouveront leurs échos dans la logique interne de cette discipline.

A priori, la littérature indique que « le football est une discipline sportive simple, aux règles faciles, ne nécessitant que peu de matériel pour être pratiquée. Celle-ci s'associe à un large éventail de pratiques diversifiées. Elle répond à des aspirations multiples qui se traduisent de différentes façons: Moins ou pas de compétitions, calendrier simplifié, pratique à effectif réduit, mixte, sur d'autres surfaces et en d'autres lieux que le stade et le terrain gazonné, dans la rue, une cour, une place, sur un parking, un champ, une plage »(20).

En bref, le football peut se pratiquer partout et par tout le monde. Un ballon réglementaire est le bien venu, mais on peut se contenter d'une balle de mousse, d'un ballon de tennis ou d'un ballon de plastiques, deux ou trois joueurs et la partie peut débuter (21). On peut même pratiquer le football à pieds nu avec un seul but et ne demandant aucune prédisposition particulière. Ce qui est à l'origine de la propagation de cette discipline à tous les coins du monde. 
- Le rôle de l'enseignant d'EPS, du dirigeant du club, de l'entraineur

Les résultats statistiques relèvent une différence significative en faveur des réponses (78\%) «Pas du tout d'accord » sur le rôle que peuvent jouer l'enseignant de l'EPS, le dirigeant de club et l'entraineur sur le choix de la discipline du football.

Autrement dit, ni l'enseignant d'EPS, ni le dirigeant du club et ni l'entraineur ne sont à l'origine du sentiment perçu quant au choix de la discipline du football, malgré la contribution de ces corps à l'évolution du sport.

Ils se trouvent actuellement en marge de toute émergence du professionnalisme sportif. Pourquoi ce désengagement? Quelle explication pourrions-nous attribuer à cela?

Il est clair que le rôle de l'école réside dans la transmission des savoirs qui légitiment le présent et le devenir de l'enfant. Cette institution est aussi un tremplin pour toutes les formes de pratique sportive scolaire et extrascolaire.

L'ensemble de ces idéaux affichés régulièrement dans les principaux textes doctrinaux et législatifs de notre pays sont largement inspirés des principes et des objectifs fixés dans la Charte Internationale de l'Éducation Physique et Sportive, notamment celle de l'Ordonnance n95-09 février 1995, relative à l'organisation et au développement du système national de la culture physique et sportive. Les établissements scolaires ont souvent été le réservoir ou on venait puiser les futurs champions.

C'était les enseignants d'EPS qui étaient derrière toutes ces opérations. Actuellement les données ont changées, les conditions ne sont plus les mêmes, le statut de l'EPS s'est de plus en plus dévalorisé. Pour l'année scolaire 2002 / 2003, le bilan de fin d'année de la Fédération Algérienne du Sport Scolaire* souligne un total de 4.032 participants avec un encadrement de 470 encadreurs et un total de 201 équipes engagées dans le cadre des compétitions scolaires, toutes disciplines confondues.

Une donnée largement insignifiante par rapport au nombre d'enfants scolarisés, qui est de 7.895.000. A la $26^{\text {éme }}$ Session Plénière de juin (2005) sur la pratique sportive scolaire et universitaire dont la thématique était " l'urgence d'une relance ",il est précisé que la norme d'encadrement en 2002 n'est que d'un enseignant d'EPS pour près de 36 élèves (alors qu'elle était sensiblement meilleure en 1974 de 1 pour 10 enfants, en raison de l'insuffisante augmentation des animateurs par rapport à l'important accroissement des effectifs scolarisés. Ainsi, La politique de la relance du sport scolaire a été une nécessité fondamentale pour la survie du sport civil. Elle a fait appel aux enseignants d'EPS par la promotion du mouvement sportif civil.

Quant au dirigeant du club, en qualité de responsable des suivis administratifs, il doit assurer la logistique de l'équipe et contribuer au bon fonctionnement du club en participant aux manifestations diverses organisées. Il fait partie prenante dans la gestion et la bonne marche du club, doit faire respecter le règlement intérieur et également faire maintenir en permanence un bon esprit sportif.

Le dirigeant doit d'une part, être doté d'une licence pour pouvoir accompagner l'équipe, être informé du calendrier des matchs, convoquer les joueurs, préparer les licences, fixer le lieu et l'heure des rendez-vous et assurer un climat favorable au travail ; et d'autre part, établir la feuille de match et éventuellement des rapports des 
compétitions, connaître les règlements et si possible, avoir des notions de premiers soins.

Au cours des matchs et des entraînements, le dirigeant du club épaule l'entraîneur, mais n'intervient pas dans le management de l'équipe. Il a toute autorité sur les joueurs pour faire appliquer les consignes de l'entraineur en son absence. Sa présence quotidienne dans le milieu sportif lui permet d'avoir une certaine connaissance de la discipline et la possibilité de communiquer avec les sportifs.

Les responsabilités de ces dirigeants et leurs contraintes administratives les ont souvent éloignés du terrain. Cet état de fait résulte de la difficulté de mener convenablement la mission de prospection, d'orientation et de conseils à l'attention des futurs joueurs.

Tout comme l'enseignant d'EPS et le dirigeant de club, l'entraineur est particulièrement la personne la plus proche des joueurs (22).

L'une des explications plausibles à ce questionnement se rapporte plus particulièrement à son engagement pour ce type de responsabilité. Néanmoins, l'accompagnement de l'enfant à toutes les formes de pratique organisée du football (au pied de l'immeuble, terrain vague, inter quartier...), dans le but de réunir des jeunes autour d'une même passion, permet à l'entraineur expert et observateur qualifié de tirer des conclusions du niveau de chacun des acteurs, en procédant à son orientation, condition nécessaire pour une pratique structurée et organisée (23).

\section{- Les infrastructures sportives}

Malgré la politique des pouvoirs publics mise en œuvre jusqu'à aujourd'hui pour la promotion et le développement du football - par la construction de centres sportifs spécialisés dont le but est de servir de tremplin aux athlètes d'élite et d'exemple aux débutants, les données statistiques en faveur des réponses $(87 \%)$ " Très peu d'accord » soulignent que les infrastructures sportives ne semblent pas être indispensables dans le choix de la discipline du football.

Quelle réponse pouvons-nous apporter à ce paradoxe?

A priori, la facilité avec laquelle cette discipline peut se pratiquer est l'une des explications plausibles sur le rôle insignifiant des infrastructures dans le choix de la discipline du football. Autrement dit, pour jouer au football, il suffit de pouvoir évoluer sur un terrain vague ou sur un espace réduit avec un ballon plus ou moins réglementaire. L'absence d'infrastructures n'influence finalement pas le choix de l'athlète à opter pour le football.

En effet, les conclusions de Henes Weisweiler reprises par Batty (24) rejoignent cette explication, démontrant que « la plupart des grands entraineurs préparent leurs joueurs sans équipements spécifiques, en les faisant juste entrainer dans des carrés ou surfaces réduites », un procédé d'entrainement largement appliqué au Brésil depuis les années 1970.

En définitive, les sentiments perçus dans le choix de la pratique du football ne semblent pas nécessairement liés aux infrastructures. 


\section{Conclusion}

Cette étude, dont l'objectif principal est la recherche de sentiments perçus dans le choix de la discipline du football, montre qu'il est impossible aujourd'hui de devenir un footballeur sans motivation personnelle et milieu sportif favorable. Compte tenu des résultats auxquels nous sommes arrivés, il est important de souligner que le choix de la discipline du football est fondamentalement liée à la recherche du statut de footballeur de haut niveau, de la célébrité et de l'argent, ainsi que le sentiment de sensation d'indépendance, du plaisir, de coopération et de la simplicité à pratiquer cette discipline sportive quitte à délaisser ses études. Par ailleurs, il a été démontré que ni l'enseignant d'éducation physique et sportive, ni les dirigeants du club ou même les entraîneurs, malgré leurs statuts particuliers et ni la disponibilité des infrastructures sportives ne sont à l'origine des sentiments perçus quant au choix de la discipline du football.

\section{Bibliographie}

1- Cazorla G., Montpetit R.-R., Prokop P., \& Cervetti J.-P., (1984), De l'évaluation des nageurs de haut niveau à la détection des talents, Spécial évaluation, INSEP (Eds.) Paris

2- Bui-xuan K., (2000), Introduction à la psychologie du sport, éd., Chiron, p.97

3- Vallerand R.J, col. , (1997), Les passions de l'âme, éd., Toronto, Canada, p. 465

4- Boiché J. (2006), Motivation autodéterminée et perception de conflit d'instrumentalité et au rôle de pratiquant sportif: relation et influence sur l'engagement et le désengagement sportif, Thèse doctorat, Université Joseph Fourier Grenoble I, sciences et géographie.

5- Blampani R., (2001), Statut du sportif, éd., Larcier, p.70

6- Margier E., (1999), La mobilité professionnelle du sportif rémunéré, éd., Van Hoecker, p.89

7- Caillot M., (1996), Sport et civilisation, éd., Harmattan, p.44

8- Gabaston G., Laconte B., (1994), Sport et télévision, éd., Harmattan, p.45

9- Baillet D., (2001), Les grands thèmes de la sociologie du sport, éd., Harmattan, p.127

10- Amram M., Andusse E., (2002), Un terrain idéal pour l'éducation, éd., ESF,p.58

11- Kerzabi M., (1996), Les championne dans le sport algérien, thèse de doctorat en sciences et technologie des APS, NP, Paris V

12- Duret P., Bobin D., (2003), Le sport en question, éd., Chiron, p.51 .

13- Raffael P., (2010), Le marché des footballeurs : réseaux et circuits dans l'économie globale, centre international d'étude et du sport, Peter Lang SA, p.119

14- Grehaigne J.F.( 1992), L'organisation du jeu en football, éd., Action, p.121-192

15- Mercier J., (1981), Football « au carrefour des méthodes », revue EPS, Paris, p.9-45

16- Sordelo J., (2004), Coaching du sportif « Sport collectif et individuel : Pour atteindre la performance », éd., Amphora, p.31. 
17- Dameron S., (2000), La dynamique relationnelle au sein d'une équipe de conception, éd.,Puf, p.340

18- Smith K., \& Caroll S., (1995), Intra et inter-organisation coopération, Academy of Management Journal, france,vol. 38, $\mathrm{n}^{\circ} 1, \mathrm{p} .7-23$.

19- Vermeulen H., (2004), Football : Entrainement à la zone, éd., Amphora, p.19.

20- Boniface, P., Rotman R.,(2002)., La terre est ronde comme un ballon: géopolitique du ballon, éd., du Seuil, p.98

21- Thepaut A.,(2004), Théorie des pratiques d'apprentissage, éd., Masson p.54.

22- Monbaerts J.F.,(1991), L'organisation du jeu en football, éd., Action, p. 23.

23- Mignon P., Hellal H., (1999), Football "jeu et société" éd., INSEP, p. 302.

24- Batty E., (1981), Le football européen, éd., Vigot, p.98

- Ordonnance n95-09 février 1995, relative à l'organisation et au développement du système national de la culture physique et sportive, Ministère de la Jeunesse et des Sports.

* Données FASS. Bilan technique année scolaire 2002-2003 Ministère de l'Education Nationale. 